\title{
Nonuniform Star Population of the LMC Cluster NGC $1978 ?$
}

\section{Kravtsov}

Sternberg Astron. Inst., 13, University Ave., Moscow 119899, Russia

\begin{abstract}
.
The LMC cluster NGC 1978 shows different properties in the two parts divided by its minor axis. These two parts have different color distributions of the core He-burning ( $\mathrm{CHB}$ ) stars and giant branch (GB) positions on the CMDs at and below the level of the CHB stars. These differences may be caused by spread in metallicity.
\end{abstract}

\section{Introduction}

The LMC shows the presence of pairs and even trios of clusters (Bhatia et al. 1991; Bica et al. 1992). A study of such objects is important to achieve understanding of the formation and evolution of star clusters. Binary nature of the LMC cluster NGC 1978 is one of the explanations of its high ellipticity (Fischer et al. 1992). We pay attention to a possible nonuniformity of its star population (see Alcaino et al. (1999) for details).

\section{Results and Conclusions}

Quadrants A and B (with $a_{\text {int }} \approx 0^{\prime} .8$ (150 pixels) and $a_{\text {ext }} \approx 3^{\prime} .3 ; e=(a-b) / a$ is equal to 0.3 ) separated by the cluster's minor axis are to the northeast of the cluster's major axis. They belong to the cluster's northwestern half (NWH) and the southeastern half (SEH), respectively. Figure 1 shows that the color distributions of CHB stars for quadrant B are more symmetrical, with a maximum near their centers, whereas the distributions for quadrant $A$ have an excess at their red ends compared to B. These features are also traced on corresponding color distributions of the whole sample of the CHB stars. Note that the visible position of the lower GB on CMDs of the cluster's SEH seem to be systematically bluer than that of the cluster's NWH. The differences discussed are apparent for colors formed using $B$ magnitudes, but are practically absent for $V-I$.

The color-position diagram (CPD) shows the above-mentioned differences on the Figure 2. In the color range $0.80<B-V<1.05$ most of the CHB and the lower GB stars (upper gians are excluded) with $X<0(\mathrm{SEH})$ are to the left of the vertical reference line in contrast to position of such stars having $X>0$ (NWH). The maximum difference in $B-V$ color between them may be as large as $0^{m} .05-0^{m} .07$.

The isochrones by Bertelli et al. (1994) with values of age and $[\mathrm{Fe} / \mathrm{H}]$ which are close to those of NGC 1978, show that the permitted age spread of a few 

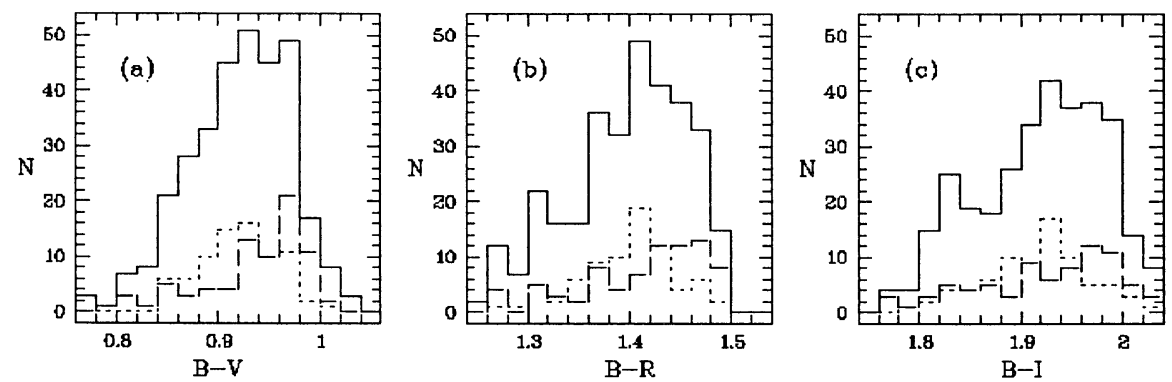

Figure 1. The color distributions (a) $B-V$, (b) $B-R$, and (c) $B-I$ of the CHB stars in different zones of NGC 1978: the dashed line for quadrant A (78 stars), the dotted line for quadrant B (77 stars), and the solid line for the whole $\left(5^{\prime} \times 5^{\prime}\right)$ cluster field (319 stars).

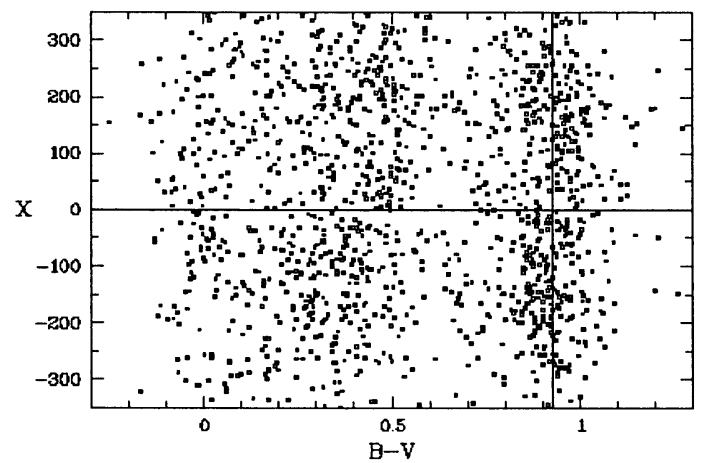

Figure 2. The CPD presents the plot of color $B-V$ versus position $(X$, in pixels) of stars along the major axis of NGC 1978. Positive and negative values of $X$ correspond to NWH and SEH of the cluster. Stars near the vertical reference line are the CHB stars and the lower giants.

tenths of a Gyr (there is no obvious variation of the turnoff magnitude) cannot cause the differences discussed. A metallicity spread leads to a scatter of the lower giants and of the CHB stars in $B-V$ to be greater than that in $V-I$ by a factor of $\sim 2.0$. The difference in metallicity between stars in NWH and SEH of NGC 1978 may be as large as $\Delta[\mathrm{Fe} / \mathrm{H}] \sim 0.1-0.2$.

\section{References}

Alcaino, G., Liller, W., Alvarado, F., et al. 1999, A\&AS, 135, 103

Bertelli, G., Bressan, A., Chiosi, C., et al. 1994, A\&AS, 106, 275

Bhatia, R. K., Read, M. A., Hatzidimitriou, D., et al. 1991, A\&AS, 87, 335

Bica, E., Claria, J. J., \& Dottori, H. 1992, AJ, 103, 1859

Fischer, P., Welch, D. L., \& Mateo, M. 1992, AJ, 104, 1086 